\title{
Study of Human Being Detection in an Indoor Environment Using Ultra Wideband Radar
}

\author{
Mohammad Amir Hassan Khausru, Mohammad Ghavami \\ Department of Electrical Engineering \\ London South Bank University \\ London SE1 0AA, UK \\ khausrum@1sbu.ac.uk, ghavamim@1sbu.ac.uk
}

\begin{abstract}
This paper describes a method of human being detection that involves eigenvalue estimation and analysis. This is a tag-free live human body detection method that looks for small changes in the reflected Ultra Wideband (UWB) signals in an indoor environment. As chest movement of the human body leaves a distinguishable effect on the reflected UWB signal hence it is an observable and detectable phenomenon that has been targeted. This experimental study also incorporates detection of the target behind the wall.
\end{abstract}

Index Terms-Human being detection, Eigenvalue estimation, Ultra Wideband signal.

\section{INTRODUCTION}

Localisation of people or assets is a well researched area in security, military and healthcare applications. In this aspect UWB Technology is emerging and becoming a suitable and well preferred area as a field of research. In our application with a view to develop a fall detection algorithm for a vulnerable sick patient living in a flat we aim to investigate this method of human body detection as a prior step.

The detection process is based on the periodic respiratory movement of the human chest that modulates the reflected UWB signal. The received signal is a frequency, phase and amplitude modulated version of the transmitted signal [1]. In our algorithm we aimed to detect this periodic change in the reflected signal and tried to find out the Time of Arrival (TOA) from the reflections in a highly cluttered indoor environment. Asith Kumar et. al [2] targeted the same problem with two different techniques called Difference Square Technique and Moving Average Reference with Discrete Fourier Transform (DFT). Target of this paper is to achieve a better resolution and more accuracy.

UWB Communication has a number of merits over other narrowband systems that facilitate currently existing localisation and positioning techniques. Its low probability of interception and detection, immunity to multipath fading, high penetration probability and fine time resolution which allows for accurate location determination makes it unique for "Ambient Assisted Living Platform" [3]. This technique can be a very good means of monitoring vulnerable older people who needs to be taken care of round the clock. Resources are always limited and it is not practical to pay for 24 hour home carers for everyone who are at a risk of a fall or other accident. UWB has recently emerged as a significant candidate for short range communications, offering the potential for short-range, through-the-wall signal pick-up and low cost networks. UWB radar can provide a complementary technology for detecting and tracking humans particularly in poor visibility or through-wall conditions and it is little affected by dust and moisture. The UWB positioning system outperformed the other technologies by being the only one to consistently achieve 3-D accuracy of less than a meter [4].

The following sections speak about Background Knowledge in section II, Algorithm Development in section III, Experimental Setup and practical experiment in section IV, Discussion in section V and finally Conclusion in section VI.

\section{BACKGROUND KNOWLEDGE}

According to Marco Baldi et. al [5], the chest movement caused by respiration and heart beat is described by the following expression:

$$
d(t)=d_{0}+m_{b} \operatorname{Sin} 2 \pi f_{b} t+m_{h} \operatorname{Sin} 2 \pi f_{h} t
$$

Where $d_{0}$ is the nominal distance between the subject and the radar antenna and $m_{b}, m_{h}\left(m_{b} \gg m_{h}\right)$ are respiration and heart beat displacement amplitudes. With assumption that the environment surrounding the subject is static, the impulse response of the channel can be modelled as -

$$
h(t, \tau)=\sum_{l_{0}}^{L} \sum_{k_{0}}^{K} \alpha_{k, l} \delta\left(\tau-T_{l}-\tau_{k, l}\right)+A \delta\left(\tau-\tau_{d}(t)\right)
$$

Where $\delta(\cdot)$ is the Dirac-delta function, $\alpha(k, l)$ is the multipath gain co-efficient, $T_{l}$ is the delay of the $l$ th cluster and $\left.\tau_{(} k, l\right)$ is the delay of the $k$ th multipath component relative to $l$ th cluster arrival time $T_{l} . \tau$ and $t$ are the fast-time and slowtime components which are in the order of nanoseconds and seconds. The sum takes into account the static contributions while the last term at the right side is related to the human chest position and its variations induced by respiration and heart beat. In particular we have,

$$
\tau_{d}(t)=\frac{2 d(t)}{c}
$$

being $c$ the velocity of light. 
Based on (2) the received signal can be written as -

$$
r(\tau)=\sum_{l} \sum_{k} \alpha_{k, l} p\left(\tau-T_{l}-\tau_{k, l}\right)+A p\left(\tau-\tau_{d}(t)\right)
$$

where $p(\tau)$ is transmitted pulse wave form. In our simulation the second derivative of the Gaussian Pulse is used as the transmitted pulse $(p(\tau))$.

\section{Algorithm DeVelopment}

The total process of live human body detection could be broken down to following steps:

- Scanning and storing of scan data

- Cross-correlating and discarding ambiguous scan

- Eliminating static clutter by making difference signals subtracting successive scans

- Segmentation and eigenvalue estimation

- Human detection and ranging

We store $N_{R}$ discrete time sequences resulting from the sampling of the received signals in a column matrix $R_{1}$. Now repeating the same process we make another column matrix $R_{2}$. Afterwards we cross correlate two successive scans (Fig. 1) and discard the scans which are below a certain level of correlation co-efficient. With this action the unnecessary ambiguous scans can be avoided.

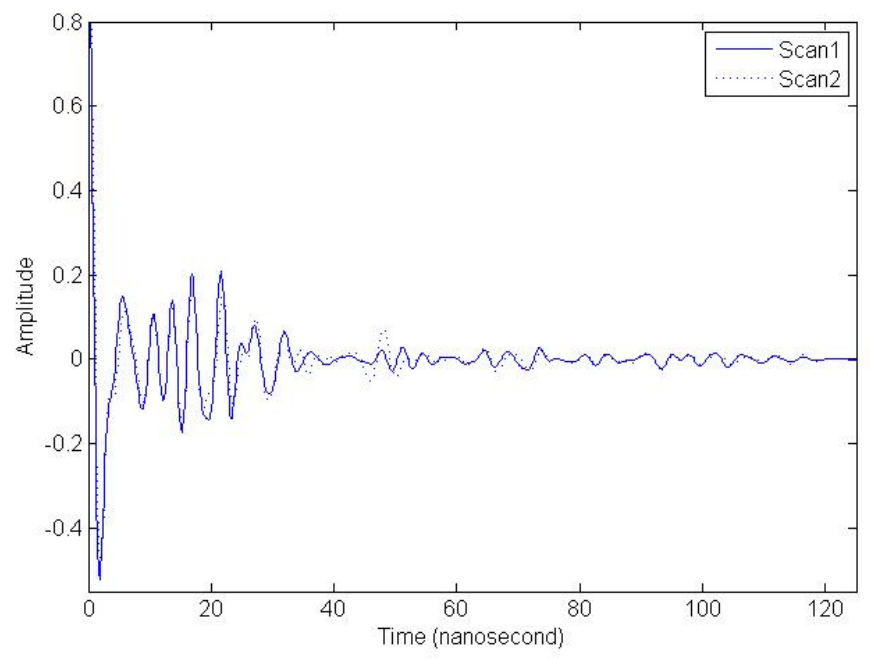

Fig. 1: Cross correlation of two successive scans in simulation.

Now the signals are normalised and to suppress the static background we subtract $R_{2}$ from $R_{1}$ that can be expressed as:

$$
\begin{aligned}
r_{d}(\tau) & =R_{1}-R_{2} \\
& =A_{1} p_{1}\left(\tau-\tau_{d}(t)\right)-A_{2} p_{2}\left(\tau-\tau_{d}(t)\right) \\
& =\Delta_{A} p\left(\tau-\tau_{d}(t)\right)
\end{aligned}
$$

which has the slight variation effect of amplitude and frequency on the incident signal due to the chest movement. Yogesh Nijsure et. al [6] used a template subtraction method to reduce the scattering of the cluttered environment which requires storing of a template signal in the absence of any human being. In [7] adaptive background subtraction method based on least mean square (LMS) algorithm was applied. Where the authors adjusted weighting coefficients in real-time according to current data in order to get the newly estimation of background . Zhu Zhang et. al [8] applied a linear least square method to estimate the stationary clutter along with the potential linear trend and subsequently subtracted from the corresponding radar data. In our algorithm we followed [2] for simplicity. Several of the above residue signals are stored in rows of a matrix A (Fig. 3). We divide this matrix into small segments along the fast-time so that we get a number of square matrixes $A_{i j} . \lambda_{i j}$ is an eigenvalue of $A_{i j}$, if there exists a non zero vector $x_{i j}$ such that $A_{i j} x_{i j}=\lambda_{i j} x_{i j}$, then $x_{i j}$ is called an eigenvector corresponding to the eigenvalue. To estimate the fast-time instant $\tau_{0}$ where the human body is located the eigenvalue corresponding to each segment is determined. We choose the square matrix for eigenvalue determination to be $9 \times 9$ or even less depending upon the condition of the environment. For any ambiguous scan the dimension of the matrix becomes smaller as the particular scan is discarded. The time at which the eigenvalue is higher indicates the presence of the target.

\section{A. Human detection}

Human detection involves the steps as follows-

a) to amplify the desired response other than the static clutter we multiply the amplitude values (eigenvalues) with the corresponding time axis values. The same action is carried out for several times. The result of this operation is described in figures $4 a, 4 b$ and $4 c$ (Fig. 4). We see that at a distance of 48 nanosecond(ns) there is a target present. The presence of human body is distinct in the following images in terms of relative intensity after multiple operations of the above technique. The first dominant peak which could occur for antenna cross-talk is suppressed due to this operation.

b) We used the "mspeaks" function of Matlab to determine the peaks and corresponding ranging information. After de-noising we filter out the peaks that are below a certain threshold level empirically set so that we get only the peaks those correspond to the moving parts of the bodies. We made a data-base where for each segment along the fast-time if there is any human body detected it will show the value 1 and 0 for no detection. Also the data-base contains the distance of the human body from the antenna when a detection is made. 
c) In this step the data base is analysed and weight of each segment is calculated. Then based on a threshold value empirically set the final decision is made whether there is a human body present or not.

We send one UWB pulse to the environment and receive the reflections during the next $180 \mathrm{~ns}$ and we define this as a "Scan". If we take 10 scans for a Scan-set and 10 Scan-sets for a detection process the total time required for the process can be calculated as follows-

1 Scan-set $=180 \times 10=1800 \mathrm{~ns}$,

1 Detection $=10$ Scan-sets $=18000 \mathrm{~ns}=18 \mu \mathrm{sec}$, for ideal case. Practically some more time is required for processing and it goes into the range of milliseconds.

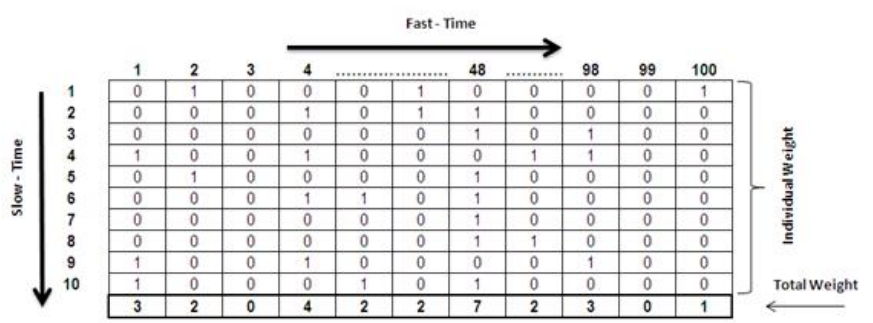

Fig. 2: Database of weights and time segments along the fasttime axis.

In the above data-base ( Fig. 2) we can look into a particular situation where we see that at a distance of $48 \mathrm{~ns}$ the decision making process puts the aggregate value of weight 7 which crosses the threshold value. As a result the human body is detected at a distance of 7.2 meter (converted from 48 ns of round-trip travel time of the signal) from the antenna according to the algorithm.

\section{B. Decision making process for human detection and ranging}

From the above data-base ( Fig. 2) putting a threshold value of the aggregate weights as 6 (empirically set) we make the decision that there is a human body present at that location. Afterwards we find the location of the body following one of the two ways as described below:

1) When making the data-base of human detection the distance is also recorded in the data base regarding each detection and the range is averaged. This average range is taken as the range of the target.

2) The particular segment along the first time axis of the data base is taken as the range of the target as the data base is made based on each time segment of the received reflected signal.

This technique could be a very good method for localising tagfree human body outperforming other existing methods [9].

\section{EXPERIMENTAL SETUP}

PulsON 400 (P400) UWB module of Time Domain has been used for the human detection process. The low power, shortrange radar works with $1.4 \mathrm{GHz} \mathrm{RF}$ band width at a low centre
TABLE I: Important aspects of the radar device(P400)

\begin{tabular}{|c|c|}
\hline Parameters & Value \\
\hline \hline Operating Band & $3.1 \mathrm{GHz}$ to $5.3 \mathrm{GHz}$ \\
\hline Centre Frequency & $4.3 \mathrm{GHz}$ \\
\hline Transmit Power & $-14.5 \mathrm{dBm}$ to $0.7 \mathrm{dBm}$ \\
\hline Noise Figure & $4.8 \mathrm{~dB}$ \\
\hline Maximum Power Consumption & 3.9 Watts \\
\hline Active (average) & 3.48 Watts \\
\hline Idle & 3.3 Watts \\
\hline
\end{tabular}

frequency of $4.3 \mathrm{GHz}$ and fully complies with the Federal Communications Commission (FCC) requirement [10].

\section{A. Human Body Detection with no Obstacle}

The experiment was performed in the office room for the research students. The environment was a highly cluttered one due to the presence of a number of desk-top computers, laptops, other equipments, wooden and metal furnitures. The experiment was carried out with distances $1 \mathrm{~m}, 1.5 \mathrm{~m}$ and $2 \mathrm{~m}$ between the antenna and the human body. We can observe that for $1 \mathrm{~m}$ separation (Fig. 5a) the human body was detected at a round-trip distance of $6.6 \mathrm{~ns}$ that is $0.99 \mathrm{~m}$ from the antenna. Fig. $5 \mathrm{~b}$ shows for $1.5 \mathrm{~m}$ separation the human body is detected at $11.8 \mathrm{~ns}$ (round-trip) that is $1.77 \mathrm{~m}$. For $2 \mathrm{~m}$ separation (Fig. 5c) the detection is displayed at $12.5 \mathrm{~ns}$ (round-trip) that is $1.875 \mathrm{~m}$.

\section{B. Behind the Barrier Application}

For behind the wall application we carried out the experiment at home. The Radar antenna was $13.5 \mathrm{~cm}$ away from a concrete wall of thickness $6.5 \mathrm{~cm}$ and the human body was 80 $\mathrm{cm}$ away from the wall (1 meter separation between human body and antenna). In Fig. 6a we see that the detection of human body is showed at a round-trip distance of $6.5 \mathrm{~ns}$ that is 0.975 meter away from the antenna. The home environment

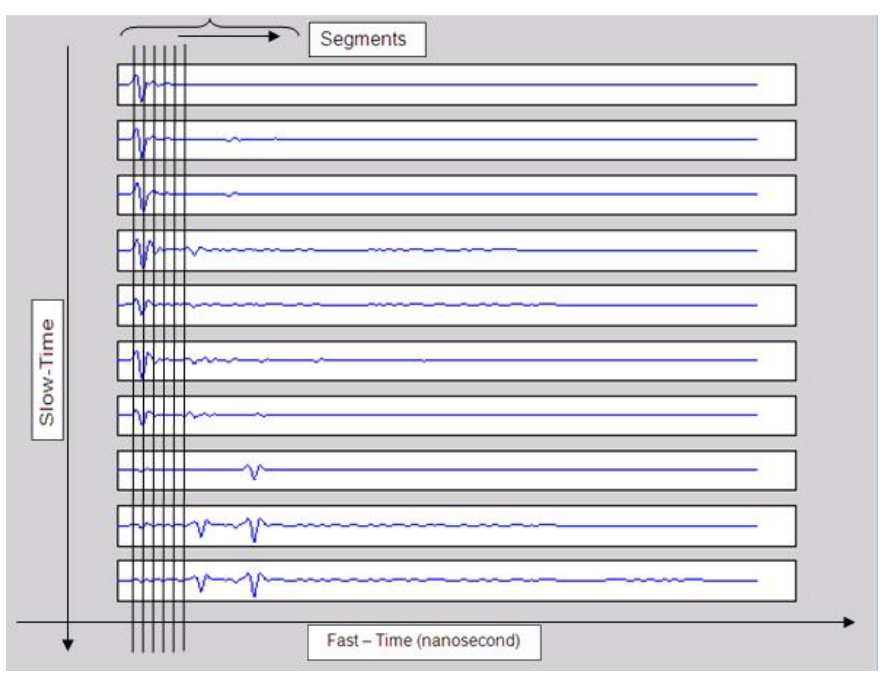

Fig. 3: Segmentation of the stored residue signals. 


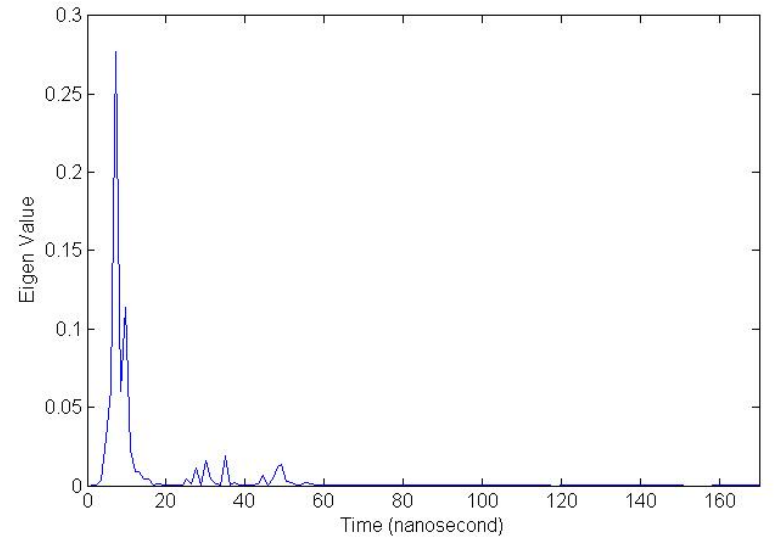

(a)

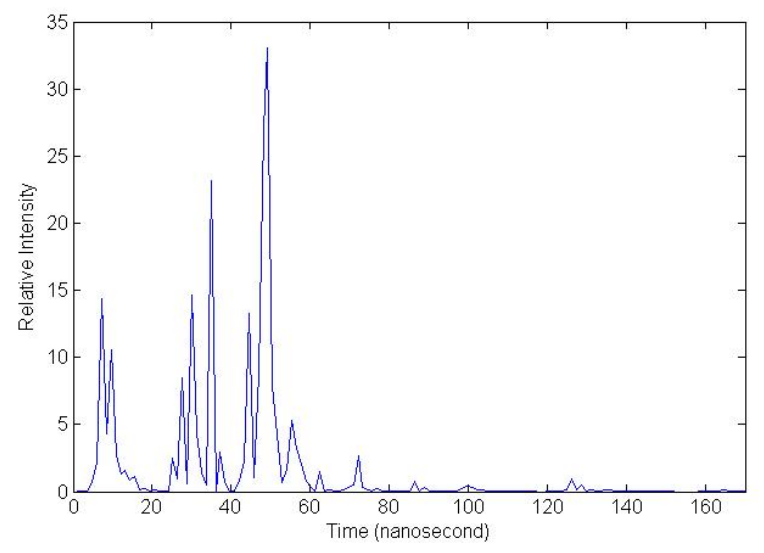

(b)

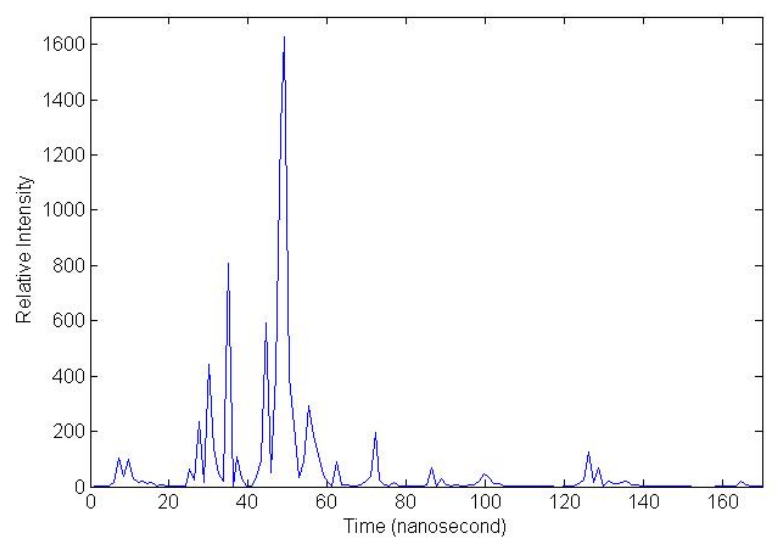

(c)

Fig. 4: In our simulation above figures show (a) plot of eigenvalues (target lies at $48 \mathrm{~ns}$ along the $\mathrm{x}$-axis), (b) amplified values of the of the movement where the target lies, (c) second time application of the same operation.

was less cluttered with no equipment or furnitures made of metals but few wooden furnitures, luggages and clothes. The door leading to the balcony was made up of glass and metals. The $6.5 \mathrm{~cm}$ wall was an extended wall inside an L-shaped

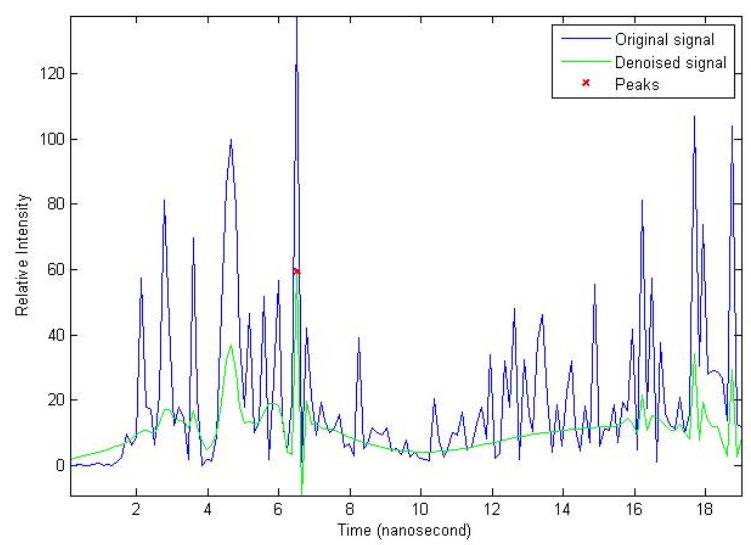

(a)

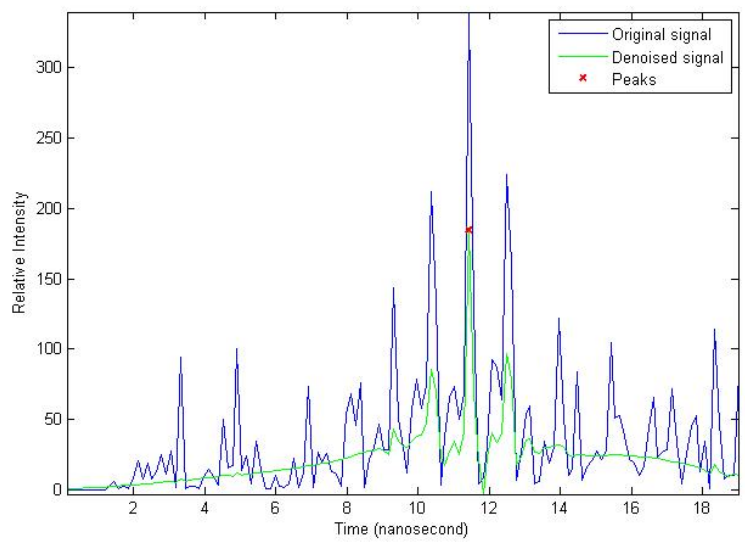

(b)

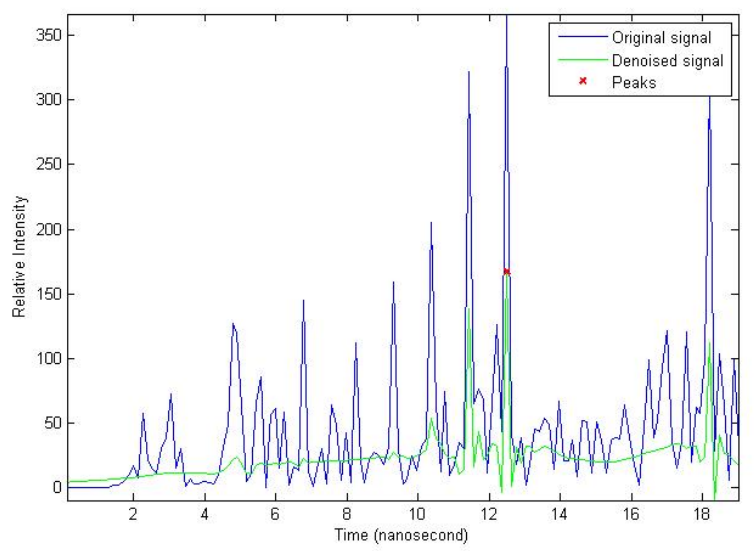

(c)

Fig. 5: Human detection in an indoor environment at distances (a) 1 meter, (b) 1.5 meter and (c) 2 meters.

double-bed room with dimensions $2.55 \mathrm{~m}, 3.80 \mathrm{~m}, 4.55 \mathrm{~m}$, $2.00 \mathrm{~m}, 1.90 \mathrm{~m}$ and $1.90 \mathrm{~m}$.

The second experiment was carried out with the same separation in the same location but this time we used a 5 $\mathrm{cm}$ thick wooden slab as the obstacle between the radar and 


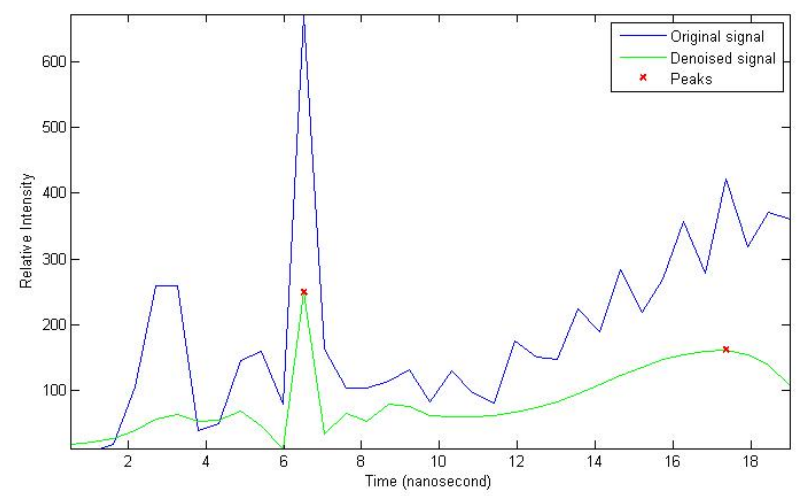

(a)

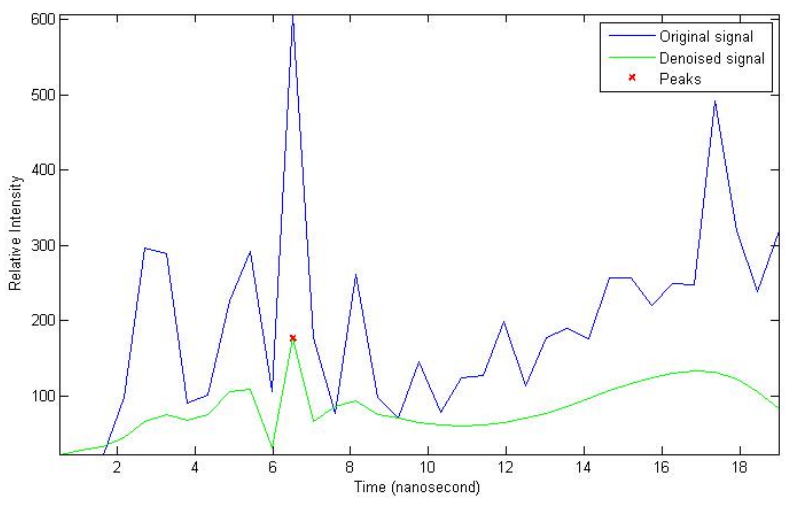

(b)

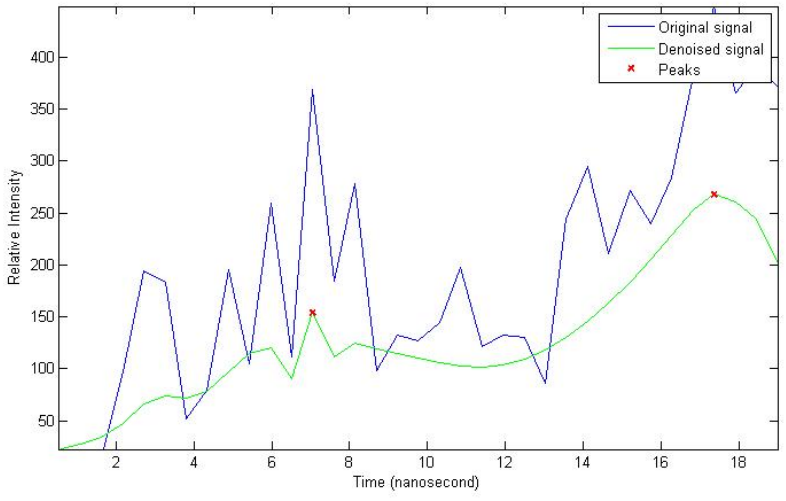

(c)

Fig. 6: For distance 1 meter behind the barrier application (a) behind a wall of thickness $6.5 \mathrm{~cm}$ (b) behind a wooden barrier of thickness $5 \mathrm{~cm}$ (c) behind a glass barrier.

the target human body. We see (Fig. 6b) that the human body is detected at the round-trip distance of $6.5 \mathrm{~ns}$ that is $0.975 \mathrm{~m}$ away from the antenna.

The third experiment (Fig. 6c) was done at the same room where the UWB radar was placed in front of a glass-door (double glazing, $2 \mathrm{~cm}$ thick) and the human being at the other side in a balcony. The distance between the radar and the target was kept the same as before but this time the differences are the radar was $38 \mathrm{~cm}$ and the target was $60 \mathrm{~cm}$ away from the glass-door. All the measurements were taken with a measuring tape. The target was detected and shown at round-trip distance of $7 \mathrm{~ns}$ that is $1.05 \mathrm{~m}$ away from the antenna.

\section{DISCUSSION}

For behind the barrier application choosing empirical threshold value is a vital factor that will reduce the number of false detection. Many of the false detections are going to be eliminated in the process of building data-base and decision making. At small distances the accuracy is much better. The method needs to be developed further for the case where there are two or more targets lies at the same distance from the antenna. This situation gives rise to the problem of missed detection, i.e., radar fails to detect a human body. Increasing the number of antennas could be one solution to the problem. Yazhou et. al talked about the solution in [11] based on the different rates of respiration for different persons. This solution will only minimise the error quantity but can not eliminate the problem completely. Because, there are many human beings who possess the same respiration rate. As UWB signals cannot pass through metals so human body behind a metal barrier will remain undetected. Hence precautions should be taken about various equipments, furnitures and their positioning inside the flat.

\section{CONCLUSION}

To locate the human body with minute details like standing up, sitting or lying down or fall down the technique described above is quite appropriate. Determining the location of the body-part like the heart or lung in a 3-D space solves the problem of determination of whether the person is standing up, sitting down, lying on bed or fall down. From the height of the moving part of the body (lungs/heart) we will be able to determine about the physical state of the person. As the method is developed to detect periodic movement of the chest, so it will not detect the unnecessary effects created by the limbs like hands and legs. By identifying the activities of daily life of a person we can detect unusual events, critical situations and trends. Wireless sensor networks can be used to measure the parameters of an individual and the immediate environment. Because of the privacy issue taking picture for image processing cannot be a suitable idea.

However this technique is not going to work for dead bodies as the detection process is based on the chest movement of the live body. So, if the patient falls and dies immediately then there will be no detection which is a draw back of the above method and needs further development.

In our experiment we didn't investigate the response for the presence of multiple human body. The human remained static without any movement during the whole experiment. But the developed technique is capable to detect for the above stated conditions. 


\section{REFERENCES}

[1] J. Li, L. Liu, Z. Zeng, and F. Liu, "Simulation and signal processing of uwb radar for human detection in complex environment." 14th International Conference on Ground Penetrating Radar (GPR), June 2012, pp. 209-213.

[2] A. Kumar, Q. Liang, Z. Li, B. Zhang, and X. Wu, "Experimental study of through-wall human being detection using ultra-wideband (uwb) radar." GC'12 Workshop: Radar and Sonar Networks, IEEE Digital Object Identifier, 2012, pp. 1455-1459.

[3] S. H. Chang, M. Wolf, and J. W. Burdick, "An mht algorithm for uwb radar-based multiple human target tracking." IEEE International Conference on Ultra-Wideband, September 2009, pp. 459-463.

[4] K. Nakamura, S. Kobayashi, H. Matsumoto, N. Koshizuka, and K. Sakamura, "Toa-based lateral distance measurement system using uwb impulse radio." IEEE 6th International Conference on Wireless and Mobile Computing, Networking and Communications, 2010, pp. 300-307.

[5] M. Baldi, F. Chiaraluce, B. Zanaj, and M. Moretti, "Analysis and simulation of algorithms for vital signs detection using uwb radars." IEEE International Conference on Ultra-Wideband (ICUWB), 2011, pp. 341-345.

[6] Y. Nijsure, W. P. Tay, E. Gunawan, F. Wen, Z. Yang, Y. L. Guan, and A. P. Chua, "An impulse radio ultra wideband system for contactless non-invasive respiratory monitoring." IEEE Transactions on Biomedical Engineering, 2013, pp. 1509-1517.

[7] H. Feng, Z. Guofu, H. Xiaotao, and Z. Zhimin, "Detection of human targets using ultra-wide band through-the-wall radar." IEEE International Conference on Microwave and Millimeter Wave Technology (ICMMT), 2010, pp. 1750-1753.

[8] Z. Zhang, X. Zhang, H. Lv, G. Lu, X. Jing, and J. Wang, "Human-target detection and surrounding structure estimation under a simulated rubble via uwb radar." IEEE Geoscience and Remote Sensing Letters, March 2013, pp. 328-331.

[9] G. M. R. I. Godaliyadda and H. K. Garg, "A time domain eigen value method for robust indoor localization." Wireless Telecommunications Symposium (WTS), 2010, pp. 1-8.

[10] A. Petroff, "A practical, high performance ultra-wideband radar platform." IEEE Radar Conference (RADAR), May 2012, pp. 0880-0884.

[11] Y. Wang, Q. Liu, and A. E. Fathy, "Simultaneous localization and respiration detection of multiple people using low cost uwb biometric pulse doppler radar sensor." Microwave Symposium Digest (MTT), June 2012, pp. 1-3. 